\title{
Adaptive Speed/Position Control of Induction Motor with Unknown Load Torque
}

\author{
Hou-Tsan Lee, ${ }^{l}$ Li-Chen $F u^{l, 2}$ and Su-Hau Hsu \\ ${ }^{1}$ Department of Electrical Engineering \\ ${ }^{2}$ Department of Computer Science and Information Engineering \\ National Taiwan University, Taipei, Taiwan, R.O.C. \\ E-mail: houtsan@cht.com.tw
}

\begin{abstract}
The paper proposes an adaptive speed/position tracking control of an induction motor subject to unknown load torque. The controller is developed based on a dynamic model obtained from the d-q-axis model (w.r.t. the stationary reference frame) of the motor under a special nonlinear coordinate transform so that either speed or position control objective can be fulfilled. The underlying design concepts is to endow the close-loop system with so-called maximal power transfer property while under lack of knowledge of some key system parameters, such as the rotor resistance, motor inertia and motor damping coefficient. To be rigorous, the proposed control scheme comes along with a thorough proof derived based on Lyapunov stability theory. The experimental results are also given to validate the effectiveness of the presented control scheme.
\end{abstract}

\section{Introduction}

The induction motor control is an important issue in both motion control and servo control applications, because the induction motor can operate in a wide-range of both torque and speed. And, their efficiency and robustness are useful features in industry.

The field oriented control (FOC) methodology is a popular control scheme of induction motor to achieve the high performance operation [1]. The control schemes based on indirect FOC are much more popular due to the advantages in applications. On the other hand, the issue of power efficiency of induction motor is well concerned nowadays. Not only the power drive is particularly considered [2] but also the power issue joint with performance is seriously taken into account [3]-[5].

In general applications, indirect vector control of induction motor is widely applied, where the rotor flux is estimated rather than being measured. This requires $a$ priori knowledge of the machine parameters, which makes the indirect vector control scheme machine dependent. Given the fact that parameters may change significantly with temperature and there are some states which are not easily acquired, design of appropriate observers becomes crucially important to the success of the control [6]. Recently, the sensorless field oriented control scheme gradually appears as a popular control method for induction motor [7]-[10]. Taking the output (speed) feedback seemingly is another effective alternative dealing with the problem with unknown resistance [8]. On the other hand, the load torque structure is also a very important knowledge for controller design to achieve high performance control.

Given the above observation, we propose a speed/position tracking control scheme based on the indirect FOC with the property of maximal power transfer to the rotor. Moreover, the proposed control scheme handles the problems with both uncertainties of rotor resistance and load torque, respectively. The system parameters of the induction motor, except its rotor resistance, and mechanical parameters, are known as mentioned previously. For rigorousness, the developed 
control scheme is thoroughly analyzed via Lyapunov stability theory, and the asymptotic convergence property is soundly proved. The experimental results are given to validate the performances.

\section{Maximal Power Transfer of Induction Motor}

In this section, we will first review the mathematical description of the operational principle of an induction motor in the following subsection. After that, we will present some important results related to the underlying concept to facilitate our subsequent controller development

As has been well known, the dynamical model of an induction motor can be simplified by a d-q-axis coordinate transformation from the original three phase representational frame to some rotational-reference frame. But to make implementation feasible, the stationary-reference frame is more popularly used. Thus, here we adopt the following group of (d-q-axis) coordinate-transformed dynamical equations of an induction motor [11]

$$
\begin{aligned}
& \dot{i}_{q s}=-\alpha_{1} i_{q s}+\alpha_{2} \lambda_{q r}-\beta p \omega_{r} \lambda_{d r}+c V_{q s} \\
& \dot{i}_{d s}=-\alpha_{1} i_{d s}+\beta p \omega_{r} \lambda_{q r}+\alpha_{2} \lambda_{d r}+c V_{d s} \\
& \dot{\lambda}_{q r}=\alpha_{3} i_{q s}-\alpha_{4} \lambda_{q r}+p \omega_{r} \lambda_{d r} \\
& \dot{\lambda}_{d r}=\alpha_{3} i_{d s}-\alpha_{4} \lambda_{d r}-p \omega_{r} \lambda_{q r} \\
& \dot{J}_{m} \dot{\omega}_{r}=T_{e}-T_{L} \\
& T_{e}=K\left(\lambda_{d r} i_{q s}-\lambda_{q r} i_{d s}\right),
\end{aligned}
$$

where the states and the parameters are defined as shown in the nomenclature.

Before we continue the control of position of the induction motors, we first make a basic assumption as shown below:

(A.1) The induction motor is assumed without saturation, hysteresis, eddy currents, and spatial flux harmonics.

Proposition 1. Under the assumptions (A.1), if the input voltages in $d-q$ frame of the voltage-fed induction motor are defined as

$$
c V_{q s}=\frac{\lambda_{d r}}{\sqrt{\lambda_{q r}^{2}+\lambda_{d r}^{2}}} V, c V_{d s}=\frac{-\lambda_{q r}}{\sqrt{\lambda_{q r}^{2}+\lambda_{d r}^{2}}} V,
$$

then the power transferred to the rotor of induction motor is maximal subject to the constraint $\left(V_{q s}^{2}+V_{d s}^{2}\right)=(V / c)^{2}$ at any time [4][5][9].

Of course, $V$ does not have to be a constant. Instead, it offers one D.O.F. (Degree of freedom) control to the system, but normally it will converge to a constant (in regulation problem) or is related to the desired output (in tracking problem) when the system approaches to the steady state.

\section{Preliminary}

To proceed with the controller design, we first introduce the observers to estimate the unmeasurable rotor flux, and the unknown rotor resistance under the following assumption:

(A.2) All the states are measurable except the rotor flux. Parameters including rotor resistance, rotor inertia, damping coefficient, and the payload coefficient are assumed unknown.

Due to Assumption (A.2), we have to build an observer and a parameter estimator to estimate the rotor flux as well as the rotor resistance. There exist various types of flux observers and parameter estimators in the literature, which have been described in [11]. Here, in this paper we adopt the observer and estimator design similar to those in [10], which is based on the stator current and rotor flux dynamics as shown below:

$$
\begin{aligned}
& L_{0} \dot{\hat{i}}_{q s}=k_{0} \tilde{i}_{q s}-\left(L_{1}+L_{n} \hat{R}_{r}\right) i_{q s}+\hat{R}_{r} \hat{\lambda}_{q r}-p L_{r} \omega_{r} \hat{\lambda}_{d r}+L_{0} c V_{q s}+u_{q}, \\
& L_{0} \hat{i}_{d s}=k_{0} \tilde{i}_{d s}-\left(L_{1}+L_{n} \hat{R}_{r}\right) i_{d s}+\hat{R}_{r} \hat{\lambda}_{d r}+p L_{r} \omega_{r} \hat{\lambda}_{q r}+L_{0} c V_{d s}+u_{d}, \\
& L_{r} \hat{\lambda}_{q r}=-\hat{R}_{r} \lambda_{q r}+p L_{r} \omega_{r} \lambda_{d r}+L_{m} \hat{R}_{r} i_{q s}, \\
& L_{r} \hat{\lambda}_{d r}=-\hat{R}_{r} \lambda_{d r}-p L_{r} \omega_{r} \lambda_{q r}+L_{m} \hat{R}_{r} i_{d s},
\end{aligned}
$$




$$
\dot{\hat{R}}_{r}=k_{R}\left[\tilde{i}_{q s}\left(\hat{\lambda}_{q r}-L_{m} i_{q s}+\frac{1}{L_{r}} \zeta_{q}\right)+\tilde{i}_{d s}\left(\hat{\lambda}_{d r}-L_{m} i_{d s}+\frac{1}{L_{r}} \zeta_{d}\right)\right],
$$

where $k_{0}, k_{R}>0$. Let the auxiliary signals $u_{q}$ and $u_{d}$ be designed as follows:

$$
u_{d}=\frac{\hat{R}_{r}}{L_{r}} \zeta_{d}+p \omega_{r} \eta_{q}, u_{q}=\frac{\hat{R}_{r}}{L_{r}} \zeta_{q}-p \omega_{r} \eta_{d},
$$

where

$$
\begin{aligned}
& \dot{\zeta}_{q}=\left(-k_{0}+k_{q 1}\right) \tilde{i}_{q s} \frac{\hat{R}_{r}}{L_{q}} \zeta_{q}+p \omega_{r} \eta_{i}, \dot{\zeta}_{d}=\left(-k_{0}+k_{d 1}\right) \tilde{i}_{d s} \frac{\hat{R}_{r}}{L} \zeta_{d}-p \omega_{r} \eta_{q}, \\
& \dot{\eta}_{q}=-\left(k_{0}+k_{q 2} \omega_{r}\right) \tilde{i}_{q s}-\frac{\hat{R}_{r}}{L_{r}} \zeta_{q}+p \omega_{r} \eta_{d}, \\
& \dot{\eta}_{d}=-\left(k_{0}+k_{d 2} \omega_{r}\right) \tilde{i}_{d s}-\frac{\hat{R}_{r}}{L_{r}} \zeta_{d}-p \omega_{r} \eta_{q},
\end{aligned}
$$

with $k_{q 1}, k_{q 2}, k_{d 1}, k_{d 2}>0$. Thus, the property of the proposed observers is summarized in the following proposition, whose proof can be found similar to that in [10].

Proposition 2. If the dynamic equations of the induction motor are described as system (1) with all the states being bounded and measurable except the rotor flux. Then, the flux observer and the rotor resistance estimator designed as in (3) can guarantee that $\hat{\lambda}_{q r} \rightarrow \lambda_{q r}, \quad \hat{\lambda}_{d r} \rightarrow \lambda_{d r}$ and $\hat{R}_{r} \rightarrow R_{r}$ as $t \rightarrow \infty$ with the system being persistently excited $(P E)$, where all the auxiliary signals being defined in (4).

For general mechanical systems, the load torque is a function of the rotor speed $\omega_{r}$ as

$T_{L}=J_{L} \dot{\omega}_{r}+\operatorname{sgn}\left(\omega_{r}\right) b_{0}+b_{1} \omega_{r}+b_{2} \operatorname{sgn}\left(\omega_{r}\right) \omega_{r}^{2}=J_{L} \dot{\omega}_{r}+f_{L}\left(\omega_{r}\right)$. This assumption is more realistic than a constant load torque. For example, it can be shown that bearings and many other viscous forces (including those encountered by cutting tools) vary linearly with speed, where the large-scale fluid systems such as pumps and fans have loads that typically vary with the square of the speed. Therefore, the mechanical load in the form aforementioned can be rearranged as $T_{L}=W_{r}^{T} \Theta$ with the constant parameter vector $\Theta=\left[\begin{array}{llll}J_{L} & b_{0} & b_{1} & b_{2}\end{array}\right]^{T}$, and the known function vector $W_{r}=\left[\begin{array}{llll}\dot{\omega}_{r} & \operatorname{sgn}\left(\omega_{r}\right) & \omega_{r} & \operatorname{sgn}\left(\omega_{r}\right) \omega_{r}^{2}\end{array}\right]^{T}$. In the sequel, we will assume that $\Theta$ is unknown, which together with the unknown constants $J_{m}$ and $B_{m}$ forms the set of unknown parameters as has been mentioned in the former assumption (A.2).

On the other hand, there exists a proper input signal $V$ such that the steady state of the system exactly achieves the purpose of speed tracking, i.e., $\omega_{r}=\omega_{d}$, and the objective of maximal power transfer (Proposition 1). To this end, we first introduce a reasonable assumption as shown below: (A.3) $x_{2}=\lambda_{q r}^{2}+\lambda_{d r}^{2}>0$, and then further simplify the dynamics shown in (1) by introducing a nonlinear state transformation given as shown in [5]. Under the transformation with Proposition 1, the dynamical equations shown in (1) can then be transformed to the following dynamic model:

$$
\begin{aligned}
& \dot{x}_{1}=-2 a_{1} x_{1}+2 a_{2} x_{3}+\frac{2 h x_{4}}{\sqrt{x_{2}}} V \\
& \dot{x}_{2}=-2 a_{4} x_{2}+2 a_{3} x_{3} \\
& \dot{x}_{3}=a_{3} x_{1}+a_{2} x_{2}-\left(a_{1}+a_{4}\right) x_{3}+p x_{5} x_{4} \\
& \dot{x}_{4}=-p x_{5} x_{3}-\left(a_{1}+a_{4}\right) x_{4}+\sqrt{x_{2}} V \\
& J \dot{x}_{5}=a_{5} x_{4}-f_{L}\left(x_{5}\right),
\end{aligned}
$$

where the parameters $a_{1}, a_{2}, a_{3}, a_{4}, a_{5}$ are defined in the nomenclature and the load structure $f_{L}\left(x_{5}\right)$ is well defined in the previous subsection. Since controller design based on a particular system structure often involves complicated algorithms which may likely lead to high implementation cost, it is highly motivated that designing a controller that can be applicable to a vast class of system structure is quite preferable.

\section{Design of Speed/Position Controllers}


In order to prove the stability of system (5) with the following proposed controller, we first present the following useful working lemma.

Lemma 1. If the state variable $x_{5}=\omega_{r}$, its derivative $\dot{x}_{5}$, and the stator currents $i_{q s}, i_{d s}$ are all bounded, then the states $x_{i}, i=1 . .5$ of system (5) are all bounded.

Proof: First, we restate the dynamic equation of $x_{2}$ from system (5) as

$$
\dot{x}_{2}=-2 a_{4} x_{2}+2 a_{3} x_{3}=-2 \alpha_{4} x_{2}+2 \alpha_{3}\left(i_{4} \lambda_{4 r}+i_{d s} \lambda_{d t}\right) \leq-\alpha_{4} x_{2}+L_{m}^{2} \alpha_{4} i_{s}^{2},
$$

where the positive parameters $\alpha_{3}, \alpha_{4}$ are defined in the nomenclature and $i_{s}^{2}=i_{q s}^{2}+i_{d s}^{2}$. Thus, $x_{2}=\lambda_{r}^{2}=\lambda_{q r}^{2}+\lambda_{d r}^{2}$ is bounded provided $i_{s}$ is bounded. Besides, from system (5), if $x_{5}$ and its derivative $\dot{x}_{5}$ are bounded, then $x_{4}$ is bounded immediately. Finally, from [5] the states $x_{1}$ and $x_{3}$, which are composed of bounded signals $i_{s}$ and $\lambda_{r}$, and hence all the states of system (5) are shown to be bounded as well.

Q.E.D.

Before we introduce the design of the controller, in order to avoid dealing with the discontinuous function $\operatorname{sgn}(x)$, we approximate it $\mathrm{y}$ the so-called sigmoid function $\operatorname{smod}(\mathrm{x})$ defined below:

$$
\operatorname{sgn}(x) \approx \operatorname{smod}(x)=\frac{e^{\gamma x}-e^{-\gamma x}}{e^{\gamma x}+e^{-\gamma x}}
$$

where $\gamma>1$ determines the slope of the function. By taking such approximation, we will be able to differentiate the payload function $f_{L}\left(\omega_{r}\right)$ for the subsequent purpose of controller design.

Theorem 1. Consider an induction motor whose dynamics are governed by system (4) with unknown load torque and rotor resistance under the assumptions (A.1) and (A.2). Given a twice-differentiable smooth desired speed trajectory $\omega_{d}$ with $\omega_{d}, \dot{\omega}_{d}$ and $\ddot{\omega}_{d}$ are all bounded, then the following control input can achieve the control objective $\omega_{r} \rightarrow \omega_{d}$ as $t \rightarrow \infty$ (i.e. $x_{5}=\omega_{r}$ will follow $\omega_{d}$ asymptotically) with the control input

$$
\begin{gathered}
V_{q s}=\frac{\lambda_{d r}}{\sqrt{\lambda_{q r}^{2}+\lambda_{d r}^{2}}} \frac{V}{c}, V_{d s}=\frac{-\lambda_{q r}}{\sqrt{\lambda_{q r}^{2}+\lambda_{d r}^{2}}} \frac{V}{c}, \text { and } \\
V=\frac{1}{\sqrt{x_{2}}}\left[\left(a_{1}+a_{4}\right) x_{4}+p x_{3} x_{5}+\dot{x}_{4 d}-a_{5} e_{5}-\rho_{2} e_{4}\right], \\
x_{4 d}=\frac{1}{a_{5}}\left(\hat{b}_{0}+\hat{b}_{1} x_{5}+\hat{b}_{2} x_{5}^{2}+\hat{J} \dot{\omega}_{d}-\rho_{1} e_{5}\right),
\end{gathered}
$$

with $\rho_{1}, \rho_{2}>0$, and $e_{5}=x_{5}-\omega_{d}, e_{4}=x_{4}-x_{4 d}$, and the

parameter estimators:

$$
\dot{\hat{\Theta}}=-e_{5} \Gamma W^{T}
$$

with $W=\left[\begin{array}{lll}\dot{\omega}_{d} \operatorname{smod}\left(x_{5}\right) & x_{5} \operatorname{smod}\left(x_{5}\right) x_{5}^{2}\end{array}\right]^{T}, \quad \Theta=\left[\begin{array}{lll}J & b_{0} & b_{1} \\ b_{2}\end{array}\right]^{T}$, while all the internal signals are kept bounded.

Proof: Omitted here, referred to Ref.[9].

In order to design the position tracking controller, we augment the system (5) with additional equation (6) as shown follow:

$$
\dot{x}_{6}=x_{5},
$$

where $x_{6}=\theta_{r}$ indicates the rotor position of the induction motor. To proceed with the design of the position controller, we first make an assumption as follow:

(A.4) The desired position command $\theta_{d} \in C^{3}$ is three-time differentiable smooth function with $\theta_{d}, \dot{\theta}_{d}, \ddot{\theta}_{d}$, and $\dddot{\theta}_{d}$ being all bounded.

Thus, if the desired speed command satisfies assumption (A.5), then the position tracking controller can be designed as stated in the following theorem.

Theorem 2. Consider the induction motor system (5) and the Eq. (6) under the Assumptions (A.I), (A2), (A.4), and (A.5). If the speed command is defined as

$$
\omega_{d}=\left\{\begin{array}{lr}
\dot{\theta}_{d}-k_{1} e_{6}, & \text { if }\left|e_{6}\right|<e_{6 m} \\
\dot{\theta}_{d}-k_{1} e_{6 m} \operatorname{smod}\left(e_{6}\right), & \text { otherwise }
\end{array}\right.
$$

with $k_{1}>0$, Then, the rotor position tracking error $e_{6}=x_{6}-\theta_{d}$ will converge to zero asymptotically with the control input $V$ being designed as in Theorem I. And, all the internal signals are thus kept bounded. 


\section{Proof:}

In order to prove the theorem completely, the proof is divided into two steps as shown below:

1) The speed command $\omega_{d}$ is a twice-differential smooth function with $\omega_{d}, \dot{\omega}_{d}$, and $\ddot{\omega}_{d}$ being all bounded due to the assumption (A.5) and the design of speed command. By Theorem 1, all the internal states $x_{1} \sim x_{5}$ are kept bounded. 2) To prove that the state $x_{6}$ is bounded and the rotor position tracking error $e_{6}$ converges to zero, we rearrange the error dynamic equation as $\dot{e}_{6}=\dot{x}_{6}-\dot{\theta}_{d}=e_{5}+\omega_{d}-\dot{\theta}_{d}$. If we choose the Lyapunov function as $V_{6}=\frac{1}{2} e_{6}^{2}$, then the time derivative of $V_{6}$ becomes

$$
\dot{V}_{6}= \begin{cases}-k_{1} e_{6}^{2}+e_{6} e_{5}, & \text { if }\left|e_{6}\right|<e_{6 m} \\ -k_{1} e_{6 m}\left|e_{6}\right|+e_{6} e_{5}, & \text { otherwise, }\end{cases}
$$

where $e_{6}=x_{6}-\theta_{d}$, and $e_{5}=x_{5}-\omega_{d}$.

As our proposition, the upper bound of $\left|e_{6}\right|$ is $e_{6 m}$. Therefore, the time derivative

$$
\dot{V}_{6} \leq-k_{1} e_{6}^{2}+e_{6} e_{5}=-2 k_{1} V_{6}+e_{6} e_{5}
$$

which readily implies boundedness of $e_{6}$ and, hence, the rotor position $\theta_{r}\left(=x_{6}\right)$. Finally, the control objective: $e_{6} \rightarrow 0$ as $t \rightarrow \infty$ is apparently achieved provided the speed error $e_{5} \rightarrow 0$ as $t \rightarrow \infty$. Moreover, as a result from step 1 and step 2, the entire internal signals $x_{i}, i=1 . .6$ are all bounded. QED.

\section{Experimental Results}

To validate the performances of the proposed controller, we hold a series experiments with a 4-ploe, 3-phase squirrel-cage induction motor which rated power 3-HP with a 1000 pulse/rev encoder. Detail parameters and specification will be found in below. The software we adopt the Simulink ${ }^{\mathrm{TM}} 3.0$ and $\mathrm{Matlab}^{\mathrm{TM}} 5.2$, we use the Simu-Drive ${ }^{\mathrm{TM}}$ to combine motor control card with Simulink $^{\mathrm{TM}} /$ Real Time Workshop ${ }^{\mathrm{TM}}$. Then we can directly apply the simulation program to proceed experiments.
$R_{s}=0.83 \Omega, R_{m}=0.53 \Omega, L_{s}=0.08601 \mathrm{H}, L_{r}=0.08601 \mathrm{H}$, $L_{m}=0.08259 \mathrm{H}, 4$ poles, rated current $8.6 \mathrm{~A}, 220 \mathrm{~V}, 60 \mathrm{~Hz}$, AC. $J_{m}$ and $B_{m}$ are assumed unknown.

The mechanical load torque is $T_{L}=$ $J_{L} \dot{\omega}_{r}+b_{s} \operatorname{sgn}\left(\omega_{r}\right)+b_{1} \omega_{r}+b_{2} \operatorname{sgn}\left(\omega_{r}\right) \omega_{r}^{2}$. In experiment, there is no load applied on the induction motor. The control gains are $\sigma_{1}=10, \sigma_{2}=10$ with $k_{d 1}=k_{d 2}=k_{q 1}=k_{q 2}=0.003$, and $k_{0}=0.01, k_{R}=200$ of the observers gains. The experimental results of both speed and position tracking are demonstrated in the following cases.

(1) The experimental results of a benchmark speed/position command

The speed/position command is like a sort of benchmark problem to validate the proposed controller. Figure 1 (or 2) shows the boundedness of estimated parameters and also the performances of the proposed controller with a benchmark speed/position command.

All the experiments are conducted without the information of the deviation of rotor resistance, the motor inertia and the damping coefficient of the induction motor. And, the parameters of load torque are unknown, either.

\section{Conclusion}

In this paper, we first develop a special nonlinear coordinate transform which makes the rotor flux norm, the electric torque and the rotor speed as individual variables $x_{2}, x_{4}$ and $x_{5}$, respectively. Then, we propose the field-oriented Lyapunov-based controllers for an induction motor to control the speed and position, respectively. And, they can also deal with both the uncertainty of rotor resistance and the unknown load torque. The experimental results validate the performances mentioned above.

\section{Reference}

[1] R. Marino, S. Peresada, and P. Tomei, "Global Adaptive Output Feedback Control of Induction Motors with Uncertain Rotor Resistance," IEEE Trans. Automat. Contr., Vol. 44, No. 5, pp. 968-983, 1999.

[2] S. K. Sul, and T. A. Lipo, "Design and Performance of a 
High-frequency Link Induction Motors Drive Operating at Unity Power Factor," IEEE Trans. Indust. Appl., Vol. 26, pp. 434-440, 1990.

[3] G. S. Kim, I. J. Ha, and M. S. Ko, "Control of Induction Motors for Both High Dynamic Performance and High Power Effiency," IEEE Trans. Indust. electron., Vol.' 39 , No.4, pp. 323-333, 1992.

[4] H. T. Lee, L. C. Fu, and H. S. Huang, "Speed Tracking Control with Maximal Power Transfer of Induction Motor," Proc. IEEE $39^{\text {th }}$ Conf. On Decision and Control, pp.925-930, 2000.

[5] H. T. Lee, J. S Chang, and L. C. Fu, "Exponential Stable Nonlinear Control for Speed Regulation of Induction Motor with Field Oriented PI-Controller," Int. J. Adap. Contr. \& Sign. Proc., Vol. 21, No.23, pp. 297-321, 2000.

[6] R. Krishnan, and A. S. Bharadwaj, "A Review of Parameter Sensitivity and Adaptation in Indirect Vector Controlled Induction Motor Drive Systems," IEEE Trans. Power Electron., Vol. 6, No.4, pp. 434-440, 1990.

[7] M. Bodson, J. Chiasson, and R. T. Novatnak, "Nonlinear Speed Observer for High-Performance Induction Motor Control," IEEE Trans. Indust. Electron., Vol. 42, No.4, pp. 434-440, 1995.

[8] R. M. Mario, S. Peresada, and P. Valigi, "Output Feedback Control of Current-Fed Induction Motors with Uncertainty of rotor resistance," IEEE Trans. Contr. Syst. Tech., Vol. 4, No. 4, pp. 336-347, 1996.

[9] H. T. Lee, and L. C. Fu, "Nonlinear Control of Induction Motor with Unknown Rotor Resistance and Load Adaptation," Proc. American Control Conference, pp. $55-59,2001$.

[10] Y. C. Lin and L. C. Fu, "Nonlinear Sensorless Indirect Adaptive Speed Control of Induction Motors with Unknown Rotor Resistance and Load", Int. J. Adap. Contr. \& Sign. Proc., Vol. 21, No.23, 2000.

[11] P. C. Krause, Analysis of Electric Machinery, McGraw-Hill, 1986.

\section{NOMENCLATURE}

$$
\begin{aligned}
& D=\left(L_{s} L_{r}-L_{m}^{2}\right) \quad \beta=L_{m} / D \quad K_{1}=3 p L_{m} / 2 L_{r} \\
& a_{1}=L_{r} R_{s} / D \quad a_{2}=L_{m} R_{s} / D \quad a_{3}=L_{m} R_{r} / D \\
& a_{4}=L_{s} R_{r} / D \quad a_{5}=L_{r} K_{t} / D \quad L_{0}=\frac{L_{r}}{L_{m}} D \\
& L_{1}=\frac{L_{r}^{2}}{L_{m}} R_{s}
\end{aligned}
$$
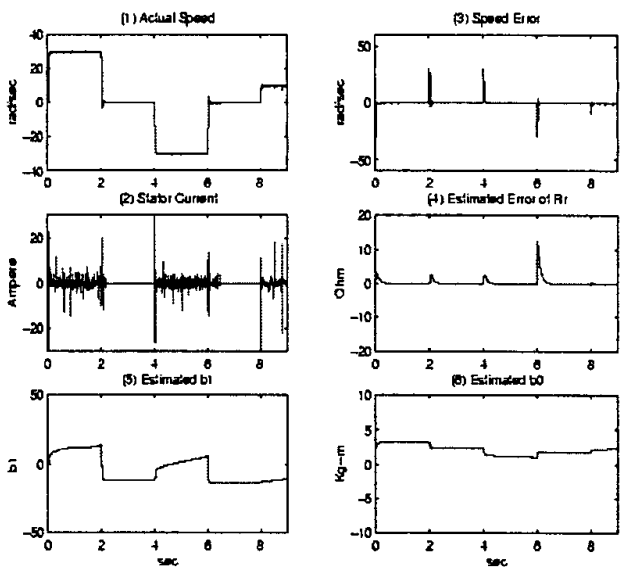

Figure 1. Experimental results of speed tracking
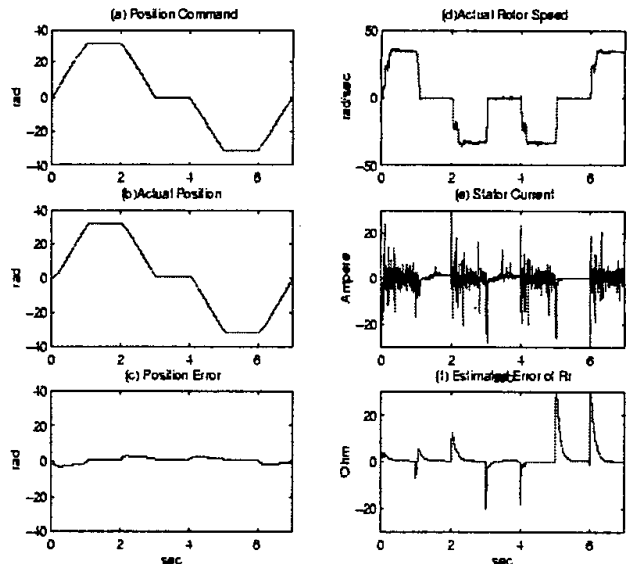

Figure 2. Experimental results of position tracking 